\title{
Solidification Defects in Additive Manufactured Materials
}

\author{
LANG YUAN ${ }^{1,2}$ \\ 1.-School of Engineering and Computing, University of South Carolina, Columbia, SC 29201, \\ USA. 2.—e-mail: langyuan@cec.sc.edu
}

Metal-based additive manufacturing processes are not limited to powder bed fusion and direct energy deposition. They have the ability to produce materials with highly customized mechanical and functional properties. Together with their freedom to fabricate complex or organic geometries, they are currently the preferred methods to make components that are difficult or impossible to manufacture by conventional means. Solidification defects, such as porosity and hot cracking, are commonly observed in metal-based additive manufacturing processes. Their existence drastically affects the mechanical and physical properties of additive manufactured materials, which in turn hinders their full potential for advanced applications. As metal 3D printers become standard equipment in most research facilities and companies, new scientific discoveries and industrial applications to understand and control solidification defects, and to establish their relationships to materials properties, are of significant interest to both academia and industry.

Established theories for the formation of solidification defects in conventional manufacturing processes, such as casting, remelting and welding, have set a solid foundation for additive manufacturing. However, it is still challenging to re-evaluate and re-confirm the underlying mechanisms due to the highly localized events in a dynamic solidification environment, where remelting, fluid flow and materials evaporation occur concurrently. In addition, defects, such as oxides, impurities and porosities, are inherent to the initial material forms, e.g., powders and wires, which are fed to fabrication processes. They also play an important role in the formation of solidification defects during the fabrication process. Recent advances in both experimental and computational modeling techniques allow in-depth understanding and the prediction of

Lang Yuan is the JOM advisor for the Solidification Committee of the TMS Materials Processing \& Manufacturing Division, and guest editor for the topic Solidification Defects in Additive Manufactured Materials in this issue. defects during rapid solidification environments. The present topic "Solidification Defects in Additive Manufactured Materials" intends to provide readers with examples of both the application of advanced computational models and the understanding of solidification defects on physical properties.

Computational modeling emerges as powerful tool to assist the exploration and provides insights to examine the mechanisms of defect formation during solidification. The first article, by Ping Jiang et al., is entitled "Fine Grains Reduce Cracking Susceptibility During Solidification: Insights from PhaseField Simulations." It employs a quantitative field phase model that simultaneously addresses solute diffusion in the solid and liquid phases, to reveal the scenes for hot cracking initiation during rapid solidification. The model shows that reducing the primary dendrite arm spacing can significantly reduce segregation in the interdendritic region and facilitate earlier coalescence of adjacent grains, which, in turn, resist solidification cracking formation. Leveraging the temperature $(T)$-solid fraction $\left(f_{s}\right)$ data calculated from the phase field models, hot cracking criteria based on the $T-f_{s}^{0.5}$ relationship is examined to further reflect the extensive coalescence in small grains. This is consistent with classic theory for hot cracking formation during solidification processes.

Another common solidification defect, porosity, can act as an initial site for cracking. Its formation is related to both initial powder conditions and process parameters, which may contain or result in entrapped gas bubbles, solidification shrinkage or lack of fusion. Controlling the porosity is also critical to the component's mechanical properties. The articl, entitled "Microstructure and Corrosion Characteristics of Selective Laser Melted 316L Stainless Steel: The Impact of Process-Induced Porosities" by Mostafa Kazemipour et al. focuses on the significance of porosities in the printed microstructure on both short-term and long-term corrosion performance, and, in the meantime, answers some questions on inconsistencies in the 
existing literature on the electrochemical stability and corrosion properties of additive-made 316L stainless steel. Anisotropic characteristics of the corrosion resistance relative to the building direction is also studied for the first time. Although the porosities do not affect the corrosion and pitting potential at the initial immersion time, their observation shows that the detrimental impacts on lowering the electrochemical stability of $316 \mathrm{~L}$ are identified at longer immersion times where large size and crevice-like pores exist.

Similarly targeting the corrosion properties, the third article, "Effects of Recycled Powder on Solidification Defects, Microstructure, and Corrosion Properties of DMLS Fabricated AlSi10Mg" by Mehran Rafieazad, confirms the degradation of the corrosion properties with the larger size and irregular shape of recycled powders. The detailed materials characterizations show that the increased volume fraction of porosities and solidification micro-cracks, and a coarsened interdendritic eutectic Si network along the melt pool boundaries, accelerates the corrosion behavior.

The following list summarizes the papers being published under the topic of "Solidification Defects in Additive Manufactured Materials". To read or download any of the papers, follow the URL http:// link.springer.com/journal/11837/71/9/page/1 to the table of contents page for the September 2019 issue (vol. 71, no. 9).

- "Fine Grains Reduce Cracking Susceptibility During Solidification: Insights from Phase-Field Simulations" by Ping Jiang, Shaoning Geng, Xinyu Shao, Gaoyang Mi, Chunming Wang, Han Wu, Chu Han, and Song Gao.

- "Microstructure and Corrosion Characteristics of Selective Laser Melted 316L Stainless Steel: The Impact of Process-Induced Porosities" by M. Kazemipour, M. Mohammadi, E. Mfoumou, and A.M. Nasiri.

- "Effects of Recycled Powder on Solidification Defects, Microstructure, and Corrosion Properties of DMLS Fabricated AlSi10Mg" by Mehran Rafieazad, Abhijit Chatterjee, and Ali Nasiri.

Publisher's Note Springer Nature remains neutral with regard to jurisdictional claims in published maps and institutional affiliations. 